\title{
Magnetic resonance imaging and analyses of tempering processes in rice kernels
}

\author{
Shyh-Shin Hwang ${ }^{\mathrm{a}}$, Yu-Che Cheng ${ }^{\mathrm{a}}$, Chen Chang ${ }^{\mathrm{b}}$, Huu-Sheng Lur ${ }^{\mathrm{c}}$, Ta-Te Lin ${ }^{\mathrm{a}, *}$ \\ ${ }^{a}$ Department of Bio-Industrial Mechatronics Engineering, National Taiwan University, Taipei 10617, Taiwan, ROC \\ ${ }^{\mathrm{b}}$ Institute of Biomedical Sciences, Academia Sinica, Taipei 11529, Taiwan, ROC \\ ${ }^{\mathrm{C}}$ Department of Agronomy, National Taiwan University, Taipei 10617, Taiwan, ROC
}

\section{A R T I C L E I N F O}

\section{Article history:}

Received 18 April 2008

Received in revised form

12 October 2008

Accepted 20 October 2008

\section{Keywords:}

Magnetic resonance imaging (MRI)

Kernel

Moisture content

Drying

\begin{abstract}
A B S T R A C T
This research involved a magnetic resonance imaging (MRI) technique that was applied to examine water distribution and migration in single rice kernels during the tempering process. The imaging experiments were performed in a Bruker 9.4T MRI system. Three-dimensional spin-echo (SE) imaging sequences were optimized by adjusting the scanning parameters of echo time (TE) and repetition time (TR) to obtain images with maximum contrast. The MR images showed that the moisture distribution in the rice kernel is non-uniform and compartmental. The embryo region exhibited much higher MR signal intensity than the starchy endosperm portion. The tempering process was analyzed with spatial-temporal signal intensities of the endosperm following the drying process of the rice kernel. The transient change of the signal intensities in the endosperm was well fitted with a double exponential function suggesting that both convection and diffusion contributed to the reduction of the moisture gradient within the rice kernel during tempering. This hypothesis was further supported by the experimental data of the insulated rice kernel whose convective mass transfer was excluded. The experimental results revealed that MR imaging of rice kernels could be used as an efficient tool to examine the mechanisms of moisture migration within cereal grains.
\end{abstract}

(c) 2009 Elsevier Ltd. All rights reserved.

\section{Introduction}

Moisture content is an important characteristic of post-harvest processing and storage of grains. Determination of moisture content is necessary in various aspects of academic research as well as practical applications. Therefore, many approaches are available to determine the moisture content of grains. Grain moisture meters based on the principles of electrical property measurement, microwave heating, infrared radiation, and near infrared spectroscopic measurements, etc., are commercially accessible and almost all methods of measurement determine the grain moisture from a batch of grain kernels. Determination of the moisture content of a single grain kernel, alternately, is relatively difficult. For the purpose of grain drying, the moisture content of the whole batch of grain kernels provides sufficient information for drying operations. However, determination of the moisture distribution and moisture migration in

\footnotetext{
* Corresponding author. Department of Bio-Industrial Mechatronics Engineering, National Taiwan University, No. 1, Roosevelt Road, Section 4, Taipei 10617, Taiwan, ROC. Tel.: +886 23366 5331; fax: +886223929416.

E-mail address: m456@ntu.edu.tw (T.-T. Lin).
}

a single grain kernel is necessary for a better understanding of rice drying processes, and thus for designing optimum drying operation.

One of the key processes in an intermittent rice drying operation is the tempering process. Tempering, a moisture relaxation process after an active drying process, is necessary to avoid a significant moisture content gradient within rice kernels that may induce undesirable fissuring of rice kernels. The objective of tempering is to equalize the moisture content by moisture migration inside the rice kernel during the process (Cnossen and Siebenmorgen, 2000; Cnossen et al., 2002). Knowledge of the tempering time is important because of the reduction in energy utilization of rice drying operation. Good design of the tempering operation will also reduce the probability of fissuring and thus ensure the good quality of the rice grains. However, most designs of tempering time are based on the accumulated experimental data and experience of the drying operation (Ponciano and Richard, 2005; Prachayawarakorn et al., 2005; Schluterman and Siebenmorgen, 2007; Shei and Chen, 1998), or analyses of simulation results based on mathematical models (Jia et al., 2002; Lu and Siebenmorgen, 1992; Yang and Jia, 2004; Yang et al., 2005).

Much research has been done with respect to understanding the phenomenon of rice fissuring and its relationship to the tempering process. Grosh and Milner (1959) used an X-ray technique to 
observe fissuring of wheat grains. Jia et al. (2001) applied both finite-element simulations and high-speed video microscopy to study the internal stress developed during drying and found the fissure appearance was consistent with theoretical predictions. In general, fissuring occurs when the moisture gradient is high enough that it produces internal stresses. There are also many other reports based on finite-element model simulations of moisture diffusion within a grain kernel to deduce the tempering time of drying processes. The criteria for tempering time determination are mainly based on avoiding a significant moisture gradient within rice kernels (Ece and Cihan, 1993; Igathinathane and Chattopadhyay, 1999; Kang and Delwiche, 1999). Although it is clear that a moisture gradient within rice kernels may exist after drying, based on computational prediction in many researches, direct measurement of moisture distribution and kinetics of the tempering process is still unable to provide validation of theoretical predictions. Measurement of moisture distribution within a grain kernel will give evidence of the causes that may induce fissuring. Nevertheless, due to the small size of grain kernels, it is relatively difficult to observe the moisture gradient during drying using methods suitable for large samples.

The recent development of a magnetic resonance imaging (MRI) technique provides a potential tool to determine moisture distribution within a grain kernel. The MRI technique provides a nondestructive means to assess both qualitative and quantitative properties of materials containing protons. The magnetic resonance signal from protons is based on the interaction between an external magnetic field and a nucleus that possesses spin (Brown and Semelka, 1995). The magnitude of the emitted signal is proportional to the number of protons present in a given volume and the relaxation times of the excited protons in the sample. MRI has been used in agriculture and food science with a number of studies in order to determine the moisture profiles of high moisture samples such as vegetables, fruits, and cereal grains. For cereal grains, highfield MRI is usually required in order to obtain sufficient spatial resolution to reveal internal moisture distribution or physicochemical properties of individual grains. Zeng et al. (1996) showed the fissuring of seed coat using the proton density MRI. Fukuoka et al. (1994) obtained water and oil distribution image of a dry soybean using chemical shift MRI. Glidewell (2006) applied both quantitative MR images and chemical shift images to detect changes in the water distribution, soluble carbohydrate and lipids of developing barley grains from anthesis to maturity. For rice grains, Takeuchi et al. (1997) obtained images of the rice grain during boiling using an MR imaging method. Frías et al. (2002) applied a constant time imaging technique to obtain the moisture content map in a central slice section of the rice kernel with a spatial resolution of $0.1 \mathrm{~mm}$. Ishida et al. (2004) applied the single point mapping imaging (SPI) technique to visualize the moisture distribution of rough rice seeds during drying. They found that the water reduction rate was greater on the outside than in the central part of the rice seeds at $50^{\circ} \mathrm{C}$ and the reduction rate of water from the rice kernels increased rapidly to $60^{\circ} \mathrm{C}$ then rose slowly above $60{ }^{\circ} \mathrm{C}$. To monitor the cooking of single rice kernels in real time, Mohorič et al. (2004) used the RARE imaging method to acquire MR images with high spatial resolution in three dimensions. Their experimental results of the MRI time series recorded during cooking compares well with gravimetric studies. A recent report by Horigane et al. (2006) moved a further step in utilizing the MRI technique to observe moisture distribution of single rice kernels. They used high-field MRI to observe water penetration into rice grains during soaking and found that the route, pattern and speed of water penetration were determined by the morphological structure, crack formation, and hardness distribution within single rice kernels.
The quality of MR images is largely dependent upon the signal intensity (S), signal-to-noise ratio (SNR), and the image resolution. For a rice kernel, the application of MRI technique faces two difficulties: small size and relatively low moisture content. To overcome these problems, this study first focused on the determination of optimum MR imaging parameters and appropriate pulse sequence for MR image acquisition. When the optimum MRI acquisition parameters are determined, experimental observation of spatial-temporal moisture distribution within single rice kernels during the tempering process can thus be performed. Hence, the specific objectives of this research include: (1) to determine optimum imaging parameters for MR images of single rice kernels based on SNR; (2) to test the feasibility of using the MRI technique to observe moisture distribution and dynamic migration within single rice kernels; and (3) to measure and quantitatively analyze dynamic moisture migration process within single rice kernels.

\section{Experimental}

\subsection{Samples}

Newly harvested rice kernels of Taikeng 8, a japonica cultivar in Taiwan, were used as test samples. The moisture contents (wet basis) of the rice kernels, determined by the standard oven method (drying at $105{ }^{\circ} \mathrm{C}$ for $72 \mathrm{~h}$ ), were approximately $29 \%$. To create a moisture gradient within rice kernels, rice kernels were dried underneath an air blower with an outlet air temperature of $55^{\circ} \mathrm{C}$ for $30 \mathrm{~min}$. The single rice kernel, at moisture content of about $20 \%$ after drying, was immediately placed into the MR spectrometer for image acquisition.

\subsection{Magnetic resonance imaging procedure}

The moisture content of rice kernels directly influences the signal intensity of MR images. Since the rice kernel is small in size and low in moisture, the signal intensity (S) and signal-to-noise ratio (SNR) are relatively low compared with other agricultural products such as fruits or vegetables. Thus, it is difficult to apply ordinary imaging sequences to image the moisture distribution of the rice kernel unless a very strong magnetic field is applied. Alternatively, a good design of pulse sequence may reveal the information of samples with low moisture content. In this study, the imaging experiments were performed on a 9.4T Bruker AMX 400 spectrometer with RF coil of $6 \mathrm{~mm}$ bore diameter and microimaging accessory. The MR spectrometer is set up in the core facility laboratory in the Institute of Biomedical Sciences, Academia Sinica, Taipei, Taiwan. A small sample holder made of plexiglass acrylic cylinder was machined for rice kernel imaging. The cylindrical sample holder has a length of $25 \mathrm{~mm}$ and a diameter of $5 \mathrm{~mm}$. The cylinder was drilled to form a bore of $4.0 \mathrm{~mm}$ diameter for the placement of the rice kernel sample. The sample holder with a rice kernel inserted was placed into a micro gradient coil with temperature maintained at $25^{\circ} \mathrm{C}$ during image acquisition. The image acquisition started $5 \mathrm{~min}$ after the sample holder was placed into the micro gradient coil.

A three-dimensional (3D) standard spin-echo pulse sequence was applied for the MR imaging of water distribution in single rice kernels. The imaging sequence was optimized by adjusting the scanning parameters of echo time (TE), repetition time (TR), number of excitation (NEX), matrix size (MATRIX), and field of view (FOV) to obtain images with good quality. Among these parameters, TE and TR were the key parameters investigated to optimize the imaging sequence. The signal-to-noise ratio (SNR) 
was used as the criterion in evaluating the image quality. SNR is expressed as:

$\mathrm{SNR}=\frac{S_{\mathrm{m}}}{B_{\mathrm{std}}}$

where $S_{\mathrm{m}}$ is the mean intensity of the rice kernel in the MR image and $B_{\text {std }}$ is the standard deviation of the background signal.

When raw magnetic resonance signals were obtained, without losing image clarity, we first converted the original 32-bit images into 8-bit images of 256 gray levels for the ease of later image processing. Commercial image processing software IMAQ Vision Builder 6 (National Instruments, USA) was used for preprocessing of the MR images. The moisture profiles of rice kernels were recorded based on the image gray level read out from the IMAQ Vision Builder software and then processed by the Microsoft Excel software for later analyses.

\section{Results and discussion}

\subsection{Optimization of imaging parameters}

The quality of MR images is largely dependent upon the signal intensity, SNR, and the image resolution. Many trial experiments were performed with various parameter settings. However, using the multi-echo sequence, we used the following imaging setup to obtain the MR images of single rice kernels: FOV $=8 \times 8 \times 4(\mathrm{~mm})$, MATRIX $=128 \times 128 \times 16, \mathrm{TE}=2.098 \mathrm{~ms}, \mathrm{TR}=250 \mathrm{~ms}, \mathrm{NEX}=3$.

To quantitatively analyze the effect of TR and TE adjustment on the quality of MR images, SNR was calculated for each image using Eq. (1). Fig. 1 shows the calculated SNR of magnetic resonance images for a rice kernel as affected by TR and TE. It is apparent that the shorter TE and longer TR result in images of high SNR such that a better image quality with less background noise was obtained. However, experimenting with longer TR requires longer image acquisition time. By considering both reasonable image acquisition time and satisfactory image quality, we chose $\mathrm{TE}=2.098 \mathrm{~ms}$ and $\mathrm{TR}=250 \mathrm{~ms}$ for most experiments in measuring moisture migration during tempering process. The duration of an acquisition was about 25 min using this imaging setup and a complete experiment lasted $7 \mathrm{~h}$.

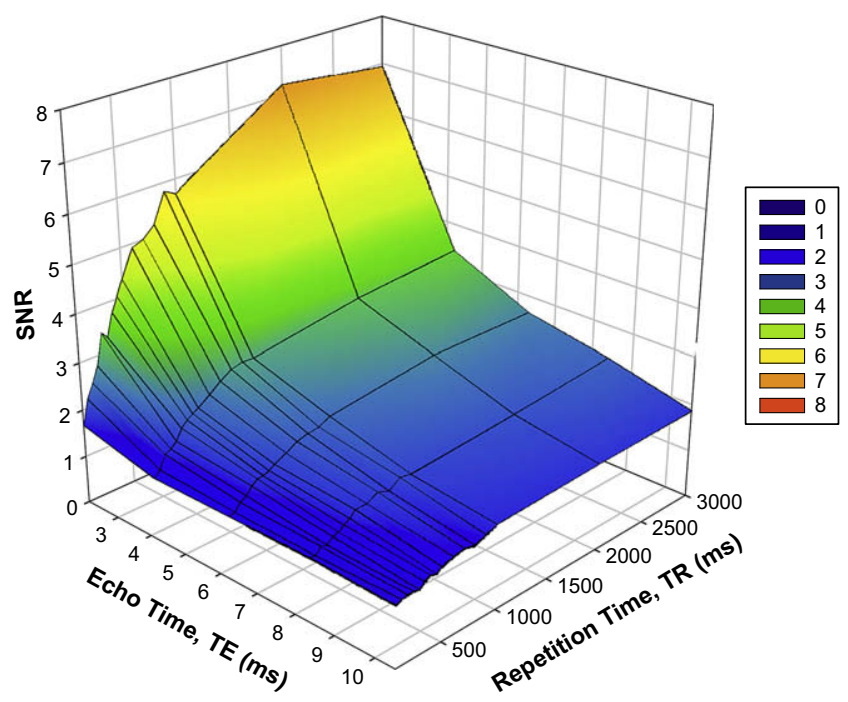

Fig. 1. The SNR of magnetic resonance images for a rice kernel as affected by the repetition time (TR) and echo time (TE).

\subsection{Moisture distribution within single rice kernels}

Experiments with continuous MR imaging were performed to observe the change in moisture distribution during the tempering of single rice kernels after an active drying process. For each rice kernel, eight longitudinal image slices out of 16 image slices from the 3D image set were extracted. Fig. 2 shows the moisture distribution of eight image slices encompassed the volume of the rice kernel: the images were acquired immediately after drying. These images have been contrast enhanced to manifest the moisture gradient within the rice kernel. Moisture gradient as well as fissuring of the rice kernel was readily observable in these image slices, especially in slices 8,9 , and 10 . This series of images demonstrates the existence of a moisture gradient after the drying process and show that the moisture gradient decreased gradually during the tempering process. The decrease of moisture gradient is more pronounced during the first $1-2 \mathrm{~h}$. The signal intensity was significantly stronger in the embryo region compared with the starchy endosperm. The aleurone layer also possessed relatively higher signal intensity. The fissures within the rice kernel were essentially perpendicular to the longitudinal axis indicating the stresses created during drying are not isotropic. This result is consistent with the finding of Jia et al. (2002) based on finiteelement simulation and high-speed microscopy imaging. They reported that, due to moisture gradient, two distinct stress zones exist inside a rice kernel during drying: a tensile zone that was closer to the surface, and a compressive zone that was closer to the center. The tensile axial stress remained at a pronounced level even after a sufficient drying duration.

The experiment was carried out for $7 \mathrm{~h}$ to observe the dynamic change of moisture distribution during the tempering process. Fig. 3 shows the time series of the contrast enhanced MR images of a rice kernel at the central slice (9th slice). The images acquired at various times depict the gradual fade out of moisture gradient during tempering. To further demonstrate the reduction of moisture gradient within a rice kernel due to tempering, we created the difference images of each rice kernel slice by subtracting the images at the end from the images at the beginning of the tempering process. Fig. 4 shows comparisons of MR images of a rice kernel before and after the tempering process. Images in column 1 and column 2 are images acquired at the beginning and at the end of the tempering process, respectively. To compare the difference between the images in column 1 and column 2, difference images were created by subtracting the images in column 2 from the images in column 1. The third column is the corresponding difference images which have been contrast enhanced with logarithmic scale transform. The difference images reflect the fact that substantial moisture gradient in the starchy endosperm was reduced during the tempering process. Fig. 4 also shows different levels of moisture gradient in different image slices. The moisture gradient in central image slices (slices 8 and 9) appear to be greater than that of outer image slices (slices 6 and 11). This experimental result indicates that the greatest moisture gradient prevails at the central part of the rice kernel after drying.

\subsection{Transient change of moisture gradient during tempering}

To further examine the moisture migration of a rice kernel during tempering, average signal intensity profiles of lateral and longitudinal sections were calculated from the MR images at different times. Fig. 5 shows the transient changes of moisture profiles within a rice kernel in both lateral and longitudinal sections. The intensity values in the figure are the average intensity values of all pixels within the corresponding $5 \times 5$ squares. It is 


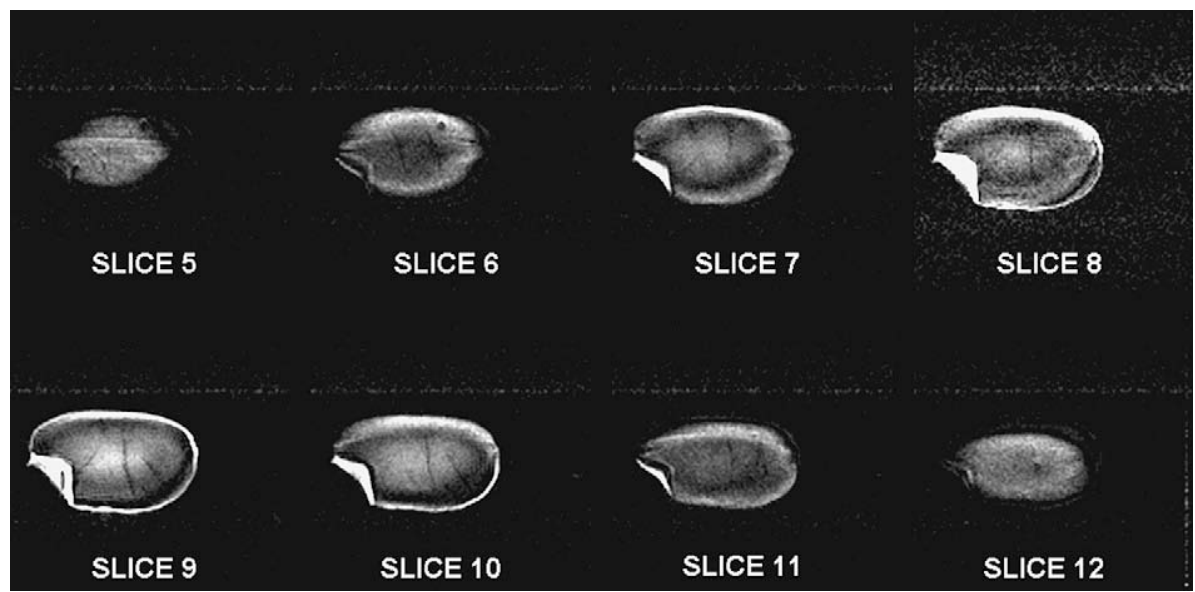

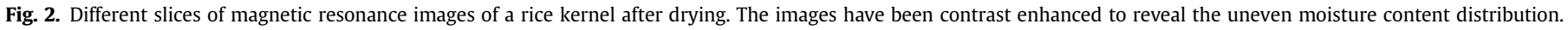

obvious that moisture content is not linear with respect to the spatial positions. The central region has significantly higher moisture content than the outer region. The difference of moisture content is less at the outer part of the endosperm. The rate of moisture reduction was faster at the earlier phase. At the end of the experiment, the moisture gradient in both lateral and longitudinal sections became much less distinct.

Fig. 6A,B show the changes of signal intensity of the central slice (9th slice) of MR images of a rice kernel during the tempering process. Transient curves of average pixel intensity of $5 \times 5$ squares at various positions within the rice kernel, in longitudinal and lateral directions, were plotted. The moisture content at the central part of the endosperm decreased with a rate much faster than that of the outer part. The central part of the endosperm is where the greatest moisture gradient existed. During tempering process, the initial signal intensity at the central part (square no. 1) decreased from 62.7 to 19.2 by the end of the experiment. The average signal intensity and standard deviation of the whole starchy endosperm part was also calculated for each image. At the beginning of the tempering process, the average signal intensity was 28.2 with a standard deviation of 14.2 while the average signal intensity at the end of the tempering process was 15.2 with a standard deviation of 8.6. The fact that the average signal intensity, which is an indicator of the overall moisture content, was not constant in this experiment suggests that moisture was lost due to convection from the kernel surface. The standard deviation of the signal intensity also decreased with time reflecting the reduction of moisture gradient during tempering.

Since the mass transport mechanism for the moisture migration of a rice kernel involves diffusion and convection processes, it is plausible to conceptually model the moisture migration as a second-order mass transport process with two different rates. Mathematically, double exponential function is a solution of a linear second-order differential equation with constant coefficient. Thus, it is reasonable to consider the double exponential function as a possible form to fit the data in Fig. 6A (Kemp et al., 2001). By fitting the transient signal intensity data at the central part of the endosperm with a double exponential function, a regression equation with $R^{2}=0.997$ was obtained whereas that to a single exponential equation is not satisfactory $\left(R^{2}=0.845\right)$ :

$S=29.27 \cdot \mathrm{e}^{-1.427 t}+31.15 \cdot \mathrm{e}^{-0.087 t}$

where $S$ is the signal intensity and $t$ is time expressed in hours. This suggests that the moisture migration is a second-order process comprised of a faster and a slower process: convection outside the kernel and diffusion inside the rice kernel. The time constant for the first exponential term corresponding to the faster process was $0.7 \mathrm{~h}$. This means that about $63.2 \%$ of the maximum moisture gradient created during the drying stage was diminished in the tempering process within $0.7 \mathrm{~h}$. The order of time constant determined in the experiment is comparable with the simulation results

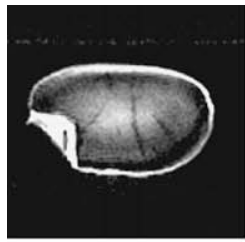

$0.0 \mathrm{Hr}$.

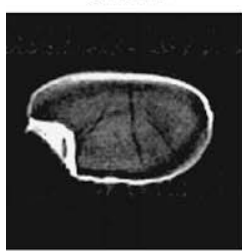

$3.0 \mathrm{Hr}$.

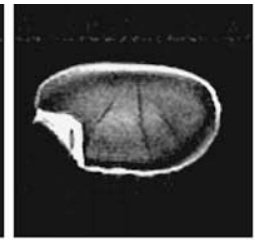

$0.5 \mathrm{Hr}$.

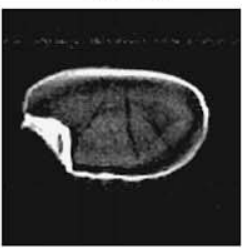

$4.0 \mathrm{Hr}$.

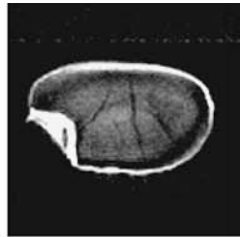

$1.0 \mathrm{Hr}$.



$5.0 \mathrm{Hr}$.

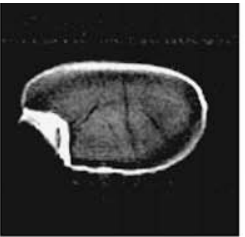

$1.5 \mathrm{Hr}$.

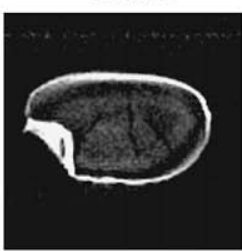

$6.0 \mathrm{Hr}$.

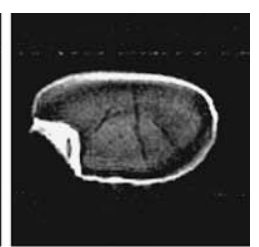

$2.0 \mathrm{Hr}$.

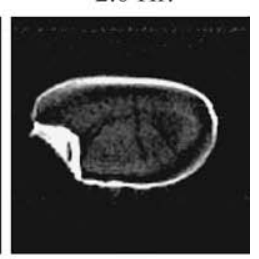

$7.0 \mathrm{Hr}$.

Fig. 3. Time series of the contrast enhanced magnetic resonance images (slice 9) of a rice kernel showing the tempering process within the rice kernel. 


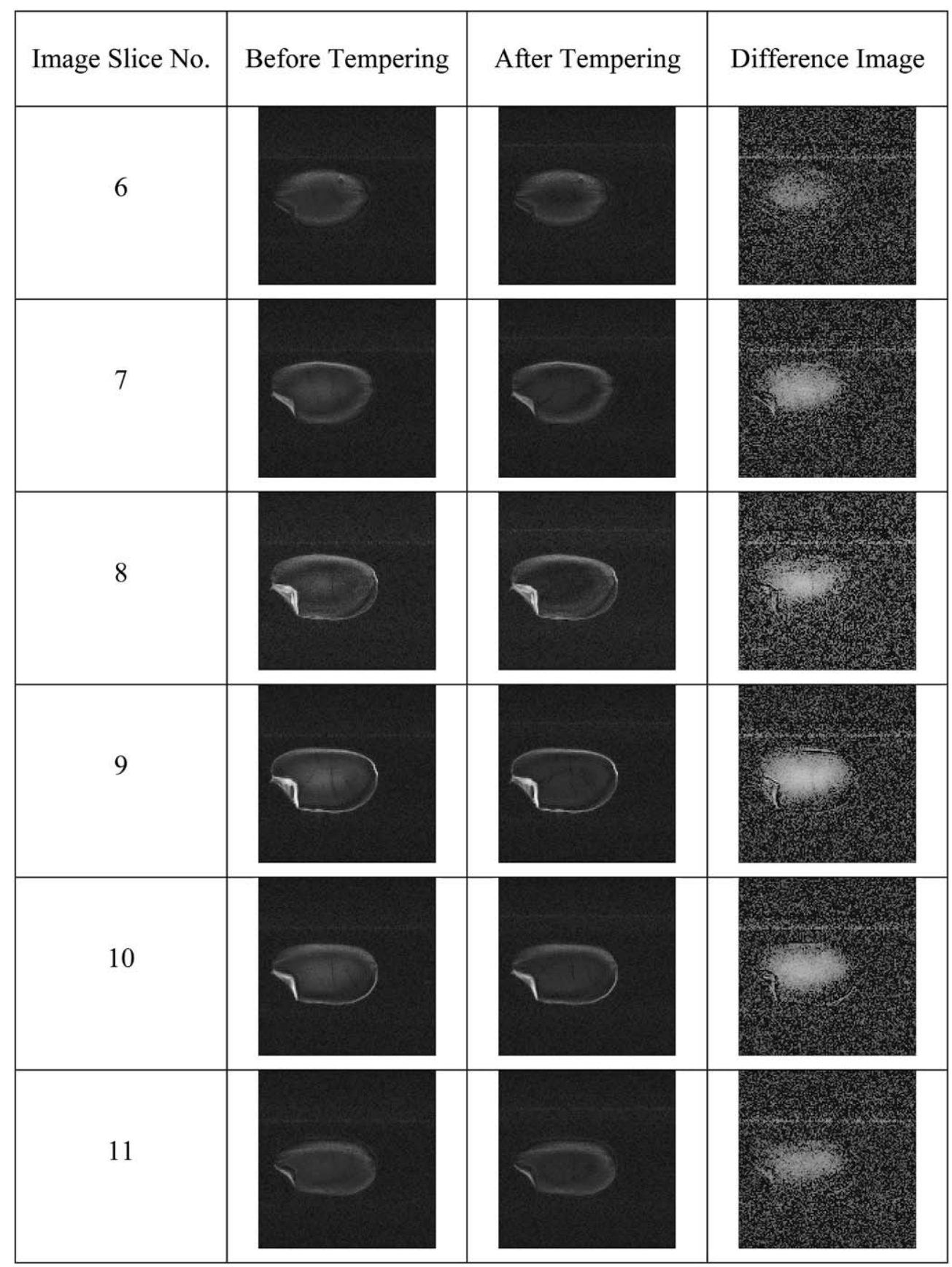

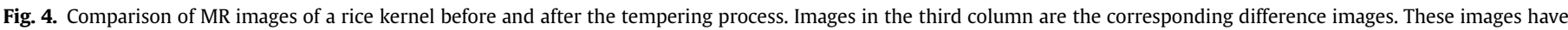
been contrast enhanced with logarithmic scale transform.

by Yang et al. (2002). They stated that a duration of about $40 \mathrm{~min}$ of tempering at $60{ }^{\circ} \mathrm{C}$ helped eliminate about $90 \%$ of the moisture gradient created inside the rice kernel during drying. The experimental results reported by Schluterman and Siebenmorgen (2007) also support our observation and analyses. Using head rice yield as an index to access tempering process, they concluded that a tempering time of $60 \mathrm{~min}$ at the drying air temperature immediately after drying was sufficient to allow intrakernel moisture content gradients to subside and thus prevent head rice yield reduction. Our experimental results based on MR image analysis provides a reference for determining the length of tempering time after an active drying process before initiating another cycle of the rice drying process. Thus, excess moisture gradient can be avoided in an intermittent rice drying operation.

\subsection{Tempering process within a sealed rice kernel}

As the temporal moisture change at the central part of a rice kernel can be modeled with a double exponential function, it is plausible to hypothesize that the kinetics of moisture migration is a second-order process. This hypothesis is consistent with the theory that two major mass transfer mechanisms, diffusion and convection, constitute the reduction of moisture gradient and removal of moisture from a rice kernel during tempering (Jia et al., 2001, 2002). Therefore, a separate experiment was designed and performed to test the hypothesis by excluding the convective mass transfer during tempering. This was done by applying the same drying condition to the rice kernel then sealing the rice kernel with paraffin wax after removing the 

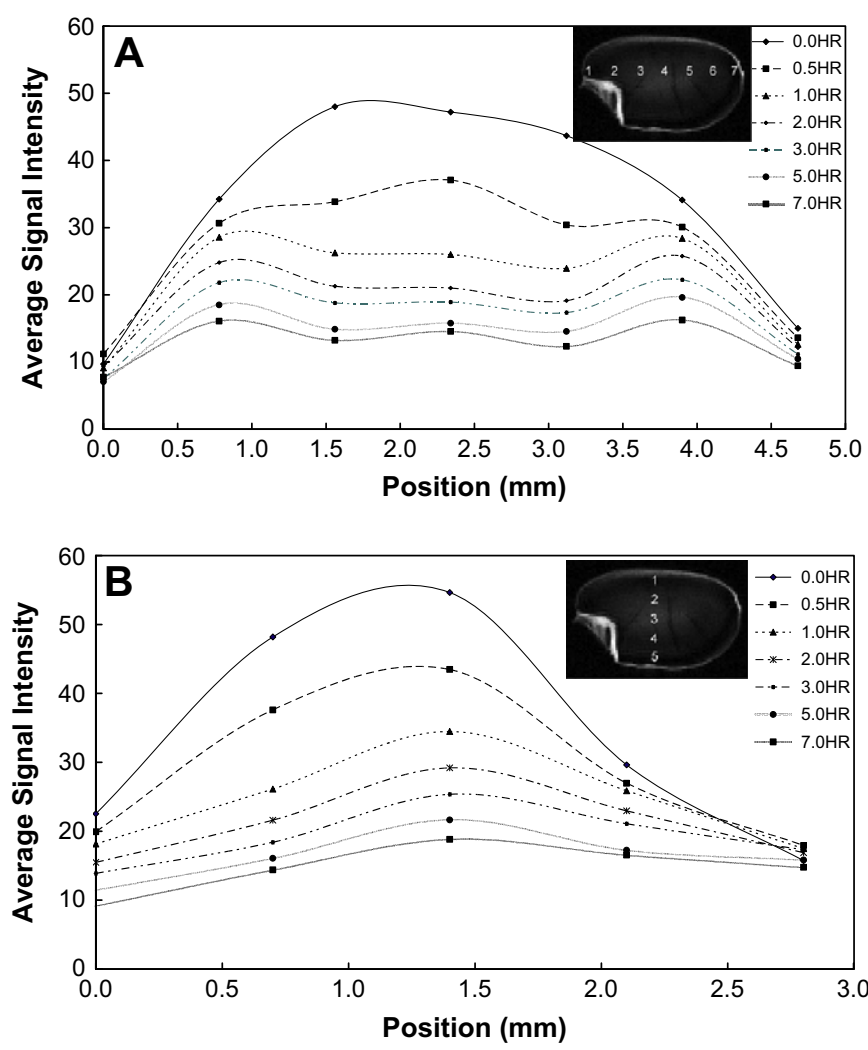

Fig. 5. Transient changes of moisture profiles within a rice kernel: (A) cross-section of the lateral axis; and (B) cross-section of the longitudinal axis.
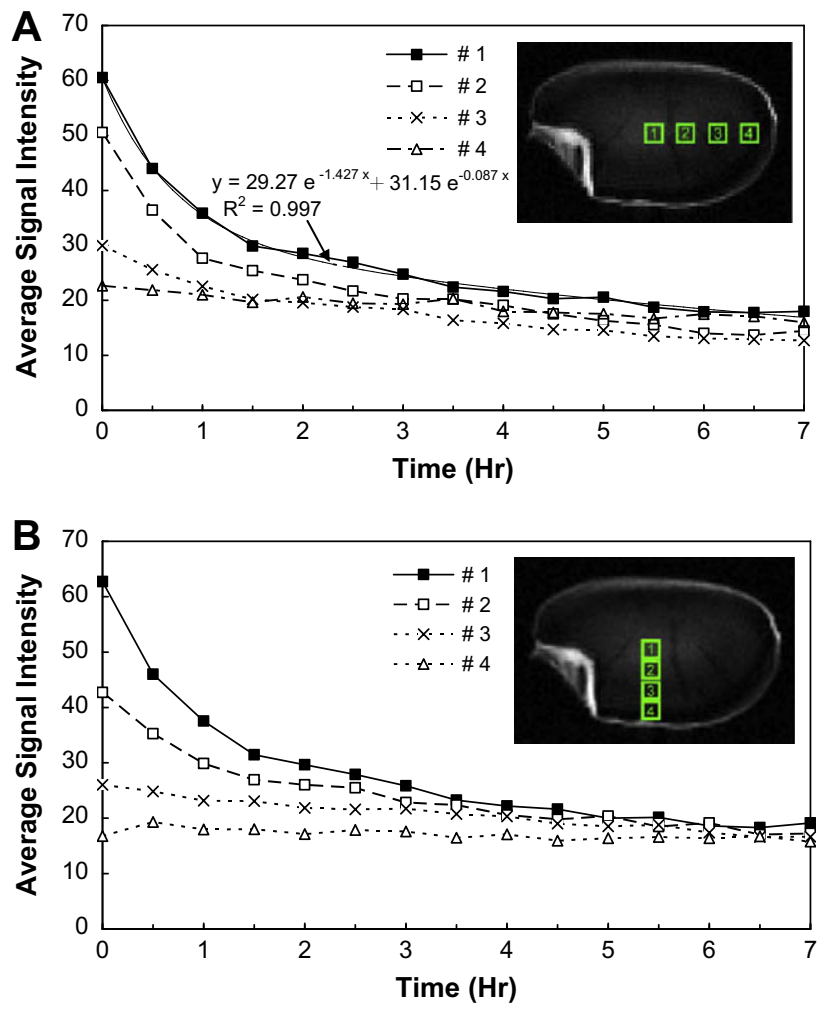

husk. The paraffin wax insulated moisture migration from the rice kernel and thus eliminated the effect of convective mass transfer.

Fig. 6C,D depict the transient change of average pixel intensity at various positions within the sealed rice kernel during tempering. Again, transient change curves of pixel intensity in longitudinal and lateral directions of a sealed rice kernel were plotted. Compared with the experimental result in Fig. 6A,B, the moisture content at the central part of the endosperm decreased with much slower rate in the sealed rice kernel. By the end of the experiment, moisture gradient still existed within the rice kernel. The transient signal intensity data can be satisfactorily fitted $\left(R^{2}=0.961\right)$ with a single exponential function (Fig. 6C) with a time constant of $7.5 \mathrm{~h}$ which is the reciprocal of the regressed coefficient 0.133 . This implies that the slower process of moisture diffusion within the rice kernel was the primary mechanism in reducing the moisture gradient within the sealed rice kernel in this experiment. The experimental data in Fig. 6 support the hypothesis that diffusion and convection constitute the reduction of moisture gradient and removal of moisture from a rice kernel during tempering.

In practical intermittent rice drying, the operation conditions may be different due to the settings of drying time, tempering time, air flow rate, air temperature, and relative humidity of air, etc. The relative contribution of convection and diffusion on the reduction of moisture gradient during tempering may be affected by these parameters. However, using the MR imaging approach to observe the spatial-temporal moisture distribution within the rice kernel as elucidated in this study, and together with appropriate model simulations, the moisture migration mechanisms can be better understood and thus practical designs for optimum drying operation can be achieved.
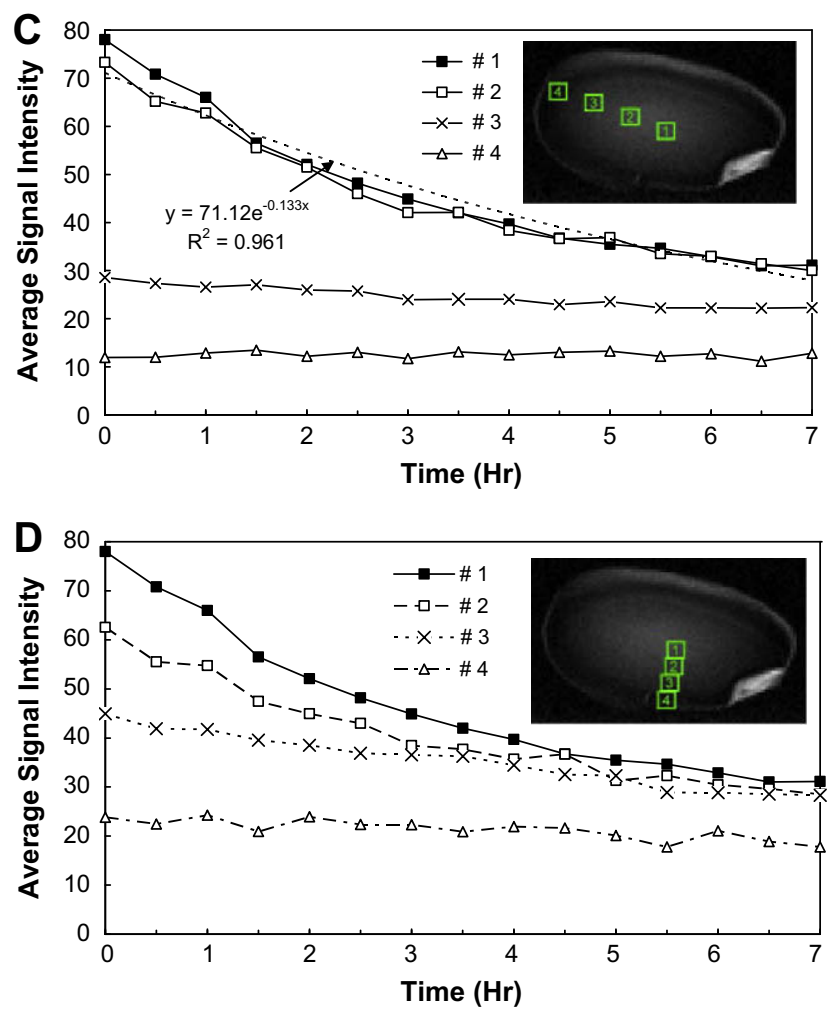

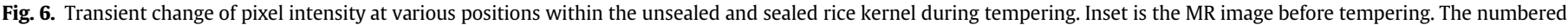

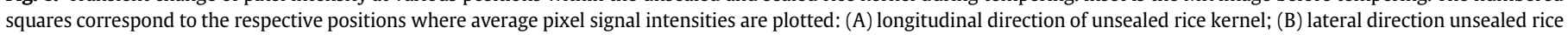
kernel; (C) longitudinal direction of sealed rice kernel; and (D) lateral direction sealed rice kernel. 


\section{Conclusions}

The multi-echo experiment was performed to acquire high-resolution MR images using various combinations of TE and TR parameters. The optimum imaging parameters were determined based on the signal-to-noise ratio and the time required for MR image acquisition. MR images of rice kernels were then acquired with optimum scanning parameters using the 3D spin-echo imaging sequence. Images with good quality were obtained using the following parameters: $\quad$ FOV $=8 \times 8 \times 4 \mathrm{~mm}, \quad$ MATRIX $=128 \times 128 \times 16$, $\mathrm{TE}=2.098 \mathrm{~ms}, \mathrm{TR}=250 \mathrm{~ms}, \mathrm{NEX}=3$. Apparent moisture variation was observed in the rice kernel. The MR images showed that the signal intensity was significantly stronger in the embryo region compared with the starchy endosperm. The moisture distributions were compared both in longitudinal and lateral sections and the analyses show that the moisture distribution is non-uniform and compartmental within the rice kernel. The dynamic moisture migration was examined with separate experiments by acquiring MR images of single rice kernel during the tempering process following drying. Experimental results indicate that the moisture content of the interior starchy endosperm was higher than that of the exterior region after drying. The moisture gradient decreased gradually during the tempering process. The moisture content at the interior part of the endosperm decreased with a rate much faster than that of the outer part. The transient signal intensity was well fitted with a double exponential function suggesting both convection and diffusion contributed to the reduction of moisture gradient during tempering. This hypothesis was further supported by the separate experiment where sealing the rice kernel with paraffin wax eliminated the effect of convection.

The experimental results and analyses in this study provide fundamental information for designing pertinent tempering time for rice drying operations. The results also reveal that the MR imaging technique is useful in investigating the dynamic moisture migration within individual rice kernel. It can be used as a reference or validation for the simulation model of moisture migration, and also as an efficient tool to examine the mechanisms of moisture migration within cereal grains.

\section{Acknowledgements}

This work was supported by the grant (NSC 95-2313-B-002-112MY3) from the National Science Council, Taiwan, ROC. The authors are grateful for technical assistance from the Functional and MicroMagnetic Resonance Imaging Center supported by the National Research Program for Genomic Medicine (NSC 94-3112-B-001-005-Y) from the National Science Council, Taiwan, ROC.

\section{References}

Brown, M.A., Semelka, R.C., 1995. MRI: Basic Principles and Applications. Wiley-Liss, Inc., New York.
Cnossen, A.G., Siebenmorgen, T.J., 2000. The glass transition temperature concept in rice drying and tempering: effect on milling quality. Transactions of the ASAE 43 (6), 1661-1667.

Cnossen, A.G., Siebenmorgen, T.J., Yang, W., 2002. The glass transition temperature concept in rice drying and tempering: effect on drying rate. Transactions of the ASAE 45 (3), 759-766.

Ece, M.C., Cihan, A., 1993. A liquid diffusion model for drying rough rice. Transactions of the ASAE 36 (3), 837-840.

Frías, J.M., Foucat, L., Bimbenet, J.J., Bonazzi, C., 2002. Modeling of moisture profiles in paddy rice during drying mapped with magnetic resonance imaging. Chemical Engineering Journal 86, 173-178.

Fukuoka, M., Watanabe, H., Mihori, T., Shimada, S., 1994. Moisture diffusion in a dry soybean seed measured using pulsed-field-gradient NMR. Journal of Food Engineering 23, 533-541.

Glidewell, S.M., 2006. NMR imaging of developing barley grains. Journal of Cerea Science $43,70-78$.

Grosh, G.M., Milner, M., 1959. Water penetration and internal fissuring in tempered wheat grains. Cereal Chemistry 36 (3), 260-273.

Horigane, A.K., Takahashi, H., Maruyama, S., Ohtsubo, K., Yoshida, M., 2006. Water penetration into rice grains during soaking observed by gradient echo magnetic resonance imaging. Journal of Cereal Science 44, 307-316.

Igathinathane, C., Chattopadhyay, P.K., 1999. Moisture diffusion modeling of drying in parboiled paddy components. Part II: bran and husk. Journal of Food Engineering 41, 89-101.

Ishida, N., Naito, S., Kano, H., 2004. Loss of moisture from harvested rice seeds on MRI. Magnetic Resonance Imaging 22, 871-875.

Jia, C.-C., Yang, W., Siebenmorgen, T.J., Bautista, R.C., Cnossen, A.G., 2001. A study of rice fissuring by finite-element simulation of internal stress combined with high-speed microscopy imaging of fissure appearance. Transactions of the ASAE 45 (3), 741-749.

Jia, C.-C., Yang, W., Siebenmorgen, T.J., Cnossen, A.G., 2002. Development of computer simulation software for single grain kernel drying, tempering, and stress analysis. Transactions of the ASAE 45 (5), 1485-1492.

Kang, S., Delwiche, S.R., 1999. Moisture diffusion modeling of wheat kernels during soaking. Transactions of the ASAE 42 (5), 1359-1365.

Kemp, I.C., Fyhr, B.C., Laurent, S., Roques, M.A., Groenwold, C.E., Tsotsas, E. Sereno, A.A., Bonazzi, C.B., Bimbenet, J.-J., Kind, M., 2001. Methods for processing experimental drying kinetics data. Drying Technology 19 (1), 15-34.

Lu, R., Siebenmorgen, T.J., 1992. Moisture diffusivity of long-grain rice components. Transactions of the ASAE 35 (6), 1955-1961.

Mohorič, A., Vergeldt, F., Gerkema, E., Jager, A.D., Duynhoven, J.V., Dalen, G.V., As, H.V., 2004. Magnetic resonance imaging of single rice kernels during cooking. Journal of Magnetic Resonance 171, 157-162.

Ponciano, S.M., Richard, P.Y., 2005. Determination of the optimum intermittent drying conditions for rough rice (Oryza sativa, L.). Lebensmittel-Wissenschaft und-Technologie 38 (2), 157-165.

Prachayawarakorn, S., Poomsa-ad, N., Soponronnarit, S., 2005. Quality maintenance and economy with high-temperature paddy-drying processes. Journal of Stored Products Research 41, 333-351.

Schluterman, D.A., Siebenmorgen, T.J., 2007. Relating rough rice moisture content reduction and tempering duration to head rice yield reduction. Transactions of the ASABE 50 (1), 137-142.

Shei, H.J., Chen, Y.L., 1998. Intermittent drying of rough rice. Drying Technology 16 (3-5), 839-851.

Takeuchi, S., Fukuoka, M., Gomi, Y., Maeda, M., Watanabe, H., 1997. An application of magnetic resonance imaging to the real time measurement of the change of moisture profile in a rice grain during boiling. Journal of Food Engineering 33, 181-192.

Yang, W., Jia, C.-C., 2004. Glass transition mapping inside a rice kernel. Transactions of the ASAE 47 (6), 2009-2015.

Yang, W., Jia, C.-C., Siebenmorgen, T.J., Howell, T.A., Cnossen, A.G., 2002. Intra-kernel moisture responses of rice to drying and tempering treatments by finite element simulation. Transactions of the ASAE 45 (4), 1037-1044.

Yang, W., Zhang, Q., Jia, C.-C., 2005. Understanding rice breakage through internal work, fracture energy, and glass transition of individual kernels. Transactions of the ASAE 48 (3), 1157-1164.

Zeng, X.S., Ruan, R.R., Fulcher, R.G., Chen, P., 1996. Evaluation of soybean seedcoat cracking during drying: Part II. Using MRI. Drying Technology 14 (7\&8), 15951623. 